\title{
Voice Controlled Home Automation Using Zigbee
}

\author{
Bhavesh C. Nandanwade ${ }^{1}$, Kinikar P. $\mathbf{I}^{2}$ \\ Student, E\&TC Dept., Daulatrao Aher College of Engg, Karad, India ${ }^{1}$ \\ Assistant Professor, E\&TC Dept., Daulatrao Aher College of Engg, Karad, India ${ }^{2}$
}

\begin{abstract}
In this paper a voice controlled wireless smart home system has been presented for elderly and disabled people. The main goal of this system is to control home appliances by using voice commands. The proposed system can recognize the voic commands, convert them into the required data format, and send the data through the wireless transmitter. In this the home appliances are controlled according the commands given by the human and the commands are recongnized by the speech recongnizer and the commands are processed by the microcontroller and loads are controlled according to the instuctions given to the microcontroller by the programmer. These commands are transmitted to the receiver from transmitter through the zigbee wireless communication zigbee works as a transreceiver here, the commands of our speech are transmitted to the receiver through zigbee from the speech recongnizer kit and the controller. The receiver side zigbee will receive the commands from the transmiter and zigbee and then to the controller and loads are operated through these commands.
\end{abstract}

Keywords: Level Shifter ,LCD Display,Micro-controller 8051, Voice Recognizer ,MAX 232 ,Relay Driver ,Zigbee.

\section{INTRODUCTION}

Voice controlled home automation systems have drawn the 7805. Therefore the output of the 7805 is constant considerable attention in the recent years. Initially, home regulated $+5 \mathrm{Vdc}$.

automation systems were designed for the people seeking luxury and sophisticated home. But, there was always a 2.ZigBee:-

need to develop home automation system for the people ZigBee is a wireless serial communication protocol,with with special needs like the elderly and the disabled.In the operating frequency of 2.4GHZ.ZigBee Home order to assist the old people and the people with disability Automation provides better operating range. With the use home automation technologies are adopting voice control of ZigBee Home Automation circuit considerable amount or voice recognition techniques. The main idea is to of power minimizing is possible and it is compatible with control and monitor home appliances by using voice future upcoming technologies so it can be easily command. The motivation behind this work is also the customized for individual requirements. same.

\section{PROPOSED SYSTEM}

\section{Micro-controller 8051:}

It is a low-power, high-performance CMOS 8-bit microcomputer with $4 \mathrm{~K}$ byte of Flash Programmable and

The basic block diagram of voice controlled home automation using zigbee is shown in the fig. 1.

Erasable Read Only Memory (PEROM).The device is manufactured using Atmel's high-density nonvolatilememory technology and is compatible with the This block diagram consist of the following essential MCS-51 $1^{\mathrm{TM}}$ instruction set and pin-out. The on-chip Flash blocks.

1. VOICE RECOGNIZER

2. MICRO-CONTROLLER 8051

3. ZIGBEE

4. MAX 232

5.Level Shifter

6.Relay Driver

\section{Power supply:} allows the program memory to be reprogrammed insystem or by a conventional non-volatile memory programmer. By combining a versatile 8-bit CPU with Flash on a monolithic chip, the Atmel AT89C51 is a powerful microcomputer,

which provides a highly flexible and cost effective solution so many embedded control applications.

\section{VOICE RECOGNITION UNIT:}

For getting +5 volts supply, the +12 volts supply from power supply output is taken. And it is given to 7805 . The minimum input to 7805 is $+7 \mathrm{Vdc}$ and Maximum input is $+35 \mathrm{vdc}$. And we are giving $+12 \mathrm{Vdc}$ as input to

The voice recognition system is completely integrated and easy to use programmable speech recognition Circuit. Programmable, in the sense that we can train the words or commands that we want the circuit to be recognized. This 
IARJSET

\author{
Vol. 4, Special Issue 2, January 2017
}

circuitry allows us to experiment with many facets of 5. Buzzer:

speech recognition technology. It has 8 bit data out which Buzzer is main indicator of the designed system through can be interfaced with any microcontroller for further which the

processing and development.

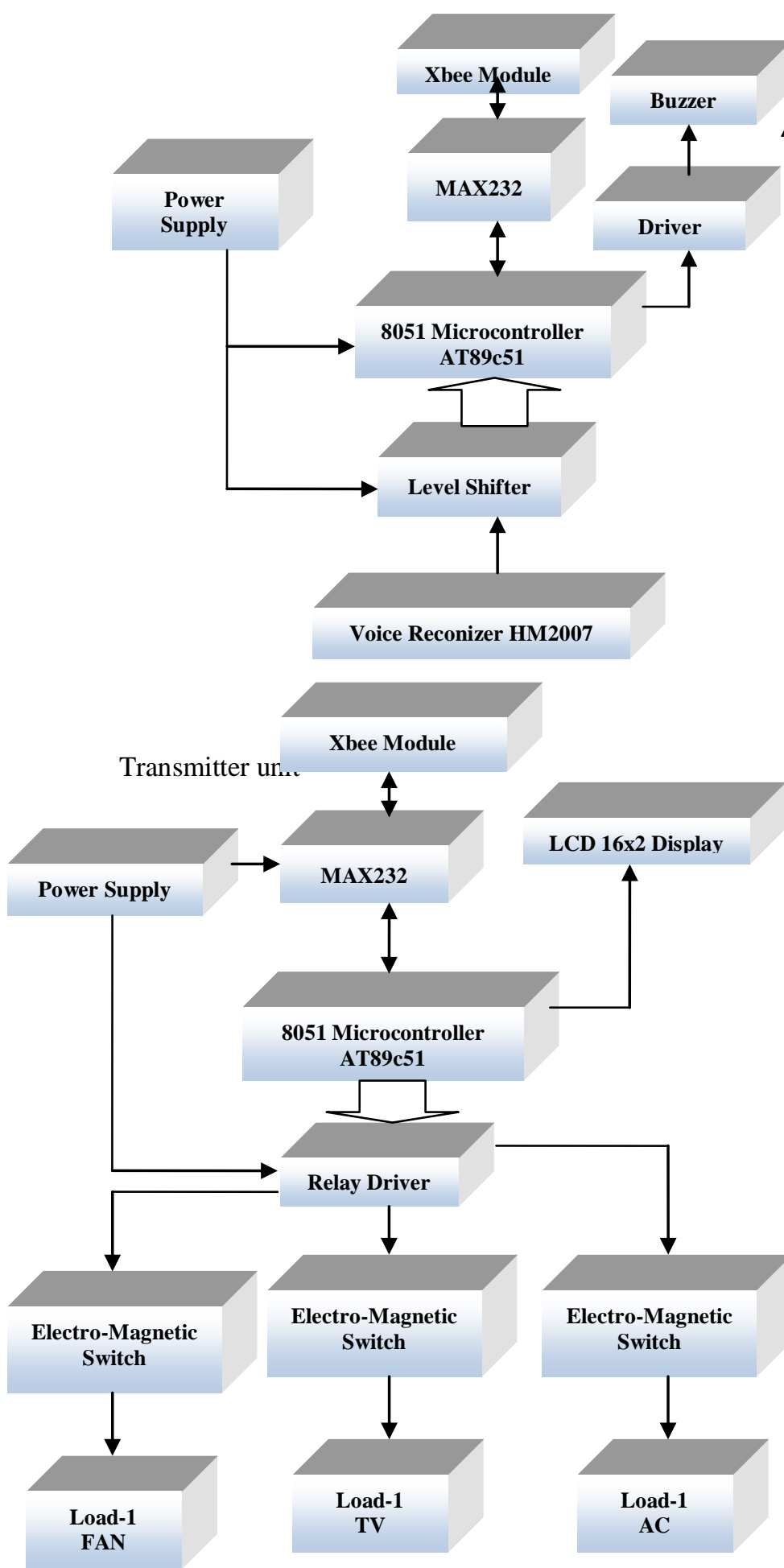

Receiver circuit

Fig. 1. System Block Diagram
Guardians of the disabled people can be alerted to check disabled person when buzzer makes a sound and take necessary care. If the patient needs then by voice command he or she may turn on the buzzer for help.

\section{System Circuit Diagram:-}
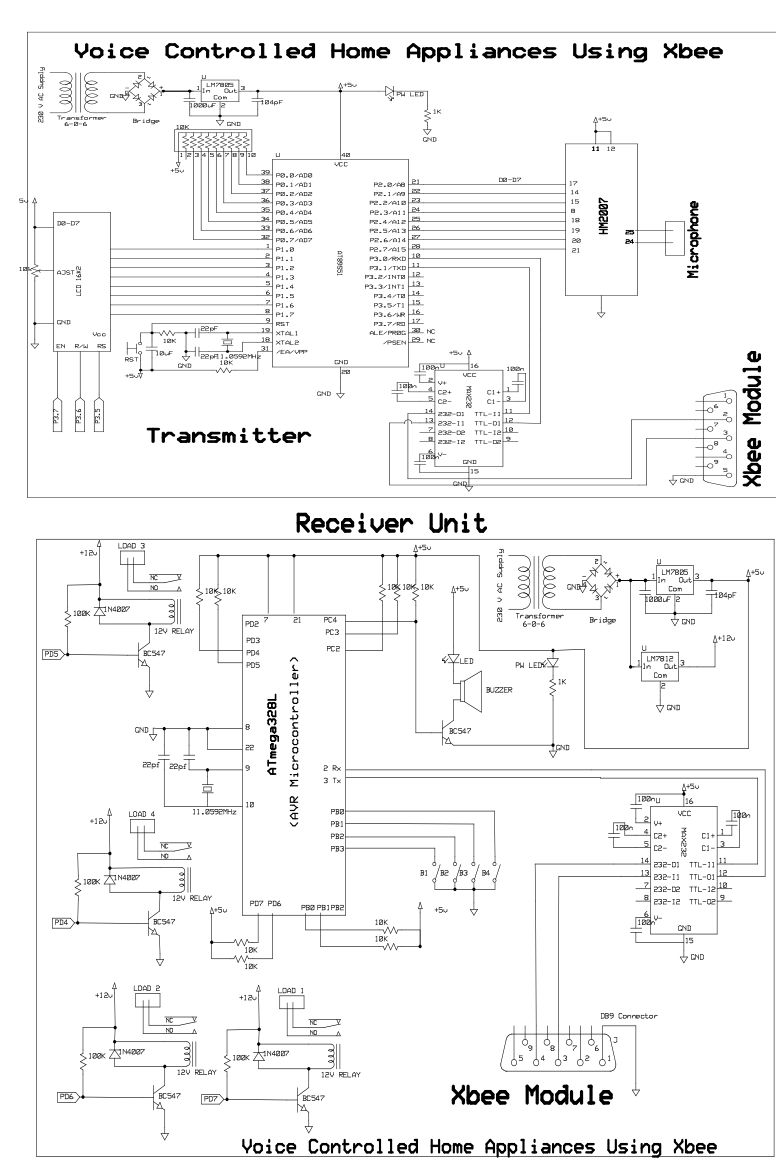

Fig.3.System Circuit Diagram

\section{REVIEW}

Home automation is used to control and monitoring of household appliances and residential house features like doors, gate,tv,fan and even the ac.This is a demonstration of how to design and buid a multi-purpose wireless system that can switch off and on any electrical household apliance depending on the voice produced.

\section{CONCLUSION}

Voice recognition Wireless Home appliances controlled system Based on ZigBee is a very useful project for the people like senior citizens and physically handicapped persons, who are unable to do different activities effectively when they are at home and need one's help to handle those tasks. With the Voice Recognition along with ZigBee network we can minimize the complexity of hardware circuitry in case of wired automation and also it 
prevent to get up and down again and again to on/off appliances.

\section{ACKNOWLEDGEMENT}

We express our sincere thanks to Ms. Kinnikar whose supervision, inspiration and valuable discussion has helped us tremendously to complete our project. His guidance proved to be the most valuable to overcome all the hurdles in the fulfillment of this Project. We grateful to Prof. Chorage P. J. for direct or indirect help in the completion of this project. Last but not least, this acknowledgement would be incomplete without rendering my sincere gratitude to all those who have helped us in the completion of this project.

\section{REFERENCES}

[1] Voice Controlled Home Automation System Based On bluetooth By Dhawan S. Thakur and Aditi Sharma

[2] ZigbeeAllianceofficialsite,[online]available:www.zigbee.org

[3] www.imageesco.com/articles/hm2007/speechrecognitiontutorial01. $\mathrm{html}$

[4] Thoraya obaid,Zigbee based voice controlledwireless smart home system, International Journal of Wireless \& Mobile Networks(IJWMN),Vol 6,No.1,February2014

[5] D.D. Chaudhary, S.P. Nayse, L.M. Waghmare, "voice controolled home automation usng Zigbee ", International Journal of Wireless \& Mobile Networks (IJWMN) Vol. 3, No. 1, February 2011.

[6] http:/airccse.org/journal/jwmn/6114ijwmn04.pdf 